\title{
Prototype for early detection of fire hazards using fuzzy logic approach and Arduino microcontroller
}

\author{
Ause Labellapansa ${ }^{1 *}$, Nesi Syafitri ${ }^{1}$, Evizal Abdul Kadir ${ }^{1}$, Rizauddin Saian ${ }^{2}$, Aqilah Saibah Binti \\ Abdul Rahman ${ }^{2}$, Marina Binti Ahmad ${ }^{2}$ and Siti Nurain Rusly ${ }^{2}$ \\ Lecturer, Department of Informatics Technology, Faculty of Engineering, Universitas Islam Riau, Riau, Indonesia ${ }^{1}$ \\ Faculty of Computer and Mathematical Sciences, Universiti Teknologi MARA, Perlis, Malaysia ${ }^{2}$
}

Received: 13-September-2018; Revised: 30-November-2018; Accepted: 30-January-2019

(C)2019 Ause Labellapansa et al. This is an open access article distributed under the Creative Commons Attribution (CC BY) License, which permits unrestricted use, distribution, and reproduction in any medium, provided the original work is properly cited.

\begin{abstract}
Fire precaution action is required in most of the buildings and any of the institution for the prevention of fire disaster. This research discusses on detection of fire hazards using sensors as the parameter that indicates for fire. The fire hazard detection system is an intelligent sensor assembled with the Arduino Microcontroller to detect flame, smoke, and gas. Most alarm system that is being used nowadays has high sensitivity to the surroundings which tends to give a false alarm. This research describes a prototype alarm system that will activate the alarm accurately based on the fuzzy logic approach that has been implemented in the system. The system uses fuzzy logic to connect with Arduino and implemented with 125 rules of fuzzy logic with three levels of output namely dangerous, potentially dangerous and not dangerous. As a result, the output of the system shows $88 \%$ accuracy.
\end{abstract}

\section{Keywords}

Fire early detection, Fuzzy logic in arduino, Prototype alarm system.

\section{Introduction}

Due to advancements in sensor technology, many tools have been created to make life safer. The main focus of this research is an early fire detection and alarm system using a fire sensor. According to the 2017 statistics provided by the department of fire and safety in Indonesia, there are 851 cases electrical fires which are caused by faulty electrical plugs and appliances, 156 cases due to stove and 1009 cases are without any explanation [1]. This research is a continuation of our previous research where we had succeeded to make a simulation of the fire hazard detector using fuzzy logic. The input from the simulation are flame, smoke, and gas, and finally, as a result, the buzzer will be activated and transmits sound of varying tones depending on the level of the danger. The level of potential danger means that the sound of the buzzer is not too fast while it becomes faster when the level is dangerous. Moreover, when the level of fire resulted in potential danger and dangerous, the water sensor will activate and or if the level of gas resulted in potential danger and dangerous the exhaust fan sensor will active.

*Author for correspondence

276
The simulation of fire hazard detector uses fuzzy logic and the Arduino microcontroller with similar inputs, but in this research, the Arduino microcontroller is limited only to activating the buzzer.

Flame, smoke and gas sensors work based on infrared light in the wavelength range of $760 \mathrm{~nm}-1100 \mathrm{~nm}$ with a detection distance of less than $1 \mathrm{~m}$ and a response time of about 15 microseconds. The MQ-2 sensor is sensitive to gas and smoke. Tin (IV) Oxide $(\mathrm{SnO} 2)$ is the main material of the sensor with low conductivity of clean air. The MQ-2 sensor is sensitive to LPG, Propane, Hydrogen, Carbon Monoxide, Methane, and Alcohol gas. The MQ-2 smoke and gas sensor sensitivity can be adjusted by rotating the resistor. This sensor is mainly used to detect gas leaks, especially at home and the industry. I-butane, propane, methane, alcohol, hydrogen, and smoke are the example of gases that can be detected by the MQ-2 sensor. There are two input voltages of the MQ-2 sensor which are $\mathrm{VH}$ and $\mathrm{VC}$. $\mathrm{VH}$ is used for the voltage on the internal heater and $\mathrm{VC}$ is the source of voltage. $\mathrm{VC}<24 \mathrm{VDC}$ and $\mathrm{VH}=5 \mathrm{~V} \pm 0,2 \mathrm{~V}$ $\mathrm{AC}$ or DC voltage is the required power supply on 
the MQ-2 sensor. Meanwhile, the MQ-2 gas sensor module is an instrument to detect smoke levels of LPG, Propane, Hydrogen, Methane and other combustible steam or any gas that causes a fire. When the air is clean, the MQ-2 sensor conductivity will be low. However, the sensor conductivity becomes high when there is a flammable gas detected in the surroundings.

MQ-2 is the cheapest sensor available and suitable for different implementations. The MQ-2 sensor provides gas content value exposed in the room and send the information to the microcontroller to be processed. This instrument is able to detect the level of gas collected in a room well because it has high sensitivity in discovering the leakage of gas [2, 3].

\section{Literature review}

\subsection{Arduino flame sensor}

Arduino is dependent on variable electronics along with applications and an open supply electronic device prototyping platform. Arduino is founded on At-mel's ATmega microcontrollers which is usually an easy yet advanced unit [4]. The ATmega 328 Microcontroller is a piece of microcontroller that can function exactly like a computer and this microcontroller will be put inside the electronic board known as Arduino Uno. The realization of the circuit electronics from simple to complex can be utilized by this device [5]. Six feet of digital can be used as a PWM (Pulse Width Modulation) signal by using Arduino Uno that has 14 feet of digital input or output. The function of the PWM signal is to regulate the motor rotation speed. The characteristics of Arduino Uno is it has a 6 pin analog input, a crystal oscillator with a clock speed of $16 \mathrm{MHz}$, a USB connection, an electrical connector, an ICSP header foot, and a reset button that serves to reset the program [2].

\subsection{Smoke sensor}

The MQ-2 smoke sensor is known as the sensor that is usually used to detect the air quality or to find out the content of the air. MQ-2 sensors are generally made of a gas-sensitive material known as carbon monoxide ( $\mathrm{CO})$. The sensor will assume that there smoke due to fire if the device detects the presence of gas in the air at certain levels [6].
To convert the Analog to Digital Converter (ADC) readings that have been converted to the voltage to be the sensor resistance, the above equation is used. The resistance of load resistance (RL) in the MQ2 sensor circuit uses $4 \mathrm{~K} 7$ in this process, therefore the resistance value of $\mathrm{RL}=4700 \Omega$ is also included in the formula [7].

\subsection{Gas sensor}

MQ-2 used to discover acetylene and hazardous gas leaks in the kitchen [8]. The gas detector will sense the existence of the gas in the environment and change it in the form of voltage level and converted by the ADC in digital form. The warning is loaded into microcontroller which will then display the results.

A mobile gas detector with an Arduino microcontroller developed by [9] to identify the risky zones where many static detectors are unsuitable or high-cost. The Arduino will manage two DC motors by using the L9110 motor driver. The HC-SR04 ultrasonic distance sensor is used to avoid collision in an analyzing area. MQ-2 will calculate the amount of gas in the surrounding. This information will be sent to the Arduino and next through a Wi-Fi module to the server. However, they still need to use the logic level converter. They stated that this is the way to get the scheme of a low-cost mobile gas detector that will be able to find a dangerous zone in analyzing the area. Thus, they presented a self-propelled robotic gas detector.

\section{Research methodology}

The research methodology is divided into two parts. The first part is related to the use of fuzzy logic that has been described on the previous research and finally explain the implementation of the Arduino fire alarm system by using the rules of previous fuzzy logic. In order to form a fuzzy set, this research uses triangle and trapezoidal functions affiliation to define the membership function. The Mamdani model approach is used as the output obtained will be in the fuzzy set. Table 1 shows the linguistic variable in each of the variables in order to create the Mamdani IF-THEN Rules and finally generated 125 rules by using Mamdani IF-THEN Rules. The fire, smoke, and gas were labelled with 0 to 4 . Meanwhile, for the grade, it was labelled from 0 to 12 .

Table 1The arrangement of the linguistic variable for each variable

\begin{tabular}{llll}
\hline Input variables & & & Output variable \\
\hline Fire & Smoke & Gas & Alarm \\
Very Large $(0)$ & Very Little $(0)$ & Very Low $(0)$ & Not Dangerous $(0-2)$ \\
Large $(1)$ & Little $(1)$ & Low $(1)$ & Potential Danger $(3-7)$ \\
\hline
\end{tabular}


Ause Labellapansa et al.

\begin{tabular}{llll}
\hline Input variables & & & Output variable \\
\hline Normal (2) & Medium (2) & Medium (2) & Dangerous $(8-12)$ \\
\hline Small (3) & Compact (3) & High (3) & \\
Very Small (4) & Very Compact (4) & Very High (4) & \\
\hline
\end{tabular}

The values will then go through the fuzzification process which is the process of changing the crisp value into a fuzzy set. After finishing the fuzzification process, the value will then check the status of the fire occurred based on the 125 of fuzzy rules. These are some of the examples of fuzzy rules:

1) If the fire is "very large" and smoke is "very little" and gas is "very low" then the alarm is "not dangerous".

2) If the fire is "very large" and smoke is "very little" and gas is "high" then the alarm is "potential danger".

3) If the fire is "very large" and smoke is "very compact" and gas is "very high" then the alarm is "dangerous".

4) If the fire is "large" and smoke is "compact" and gas is "medium" then the alarm is "potential danger".

5) If the fire is "large" and smoke is "compact" and gas is "very high" then the alarm is "dangerous".

6) If the fire is "normal" and smoke is "very little" and gas is "very low" then the alarm is "not dangerous".

7) If the fire is "small" and smoke is "medium" and gas is "very low" then the alarm is "potential danger".

8) If the fire is "small" and smoke is "medium" and gas is "high" then the alarm is "dangerous".

9) If the fire is "very small" and smoke is "very compact" and gas is "high" then the alarm is "dangerous".

10) If the fire is "very small" and smoke is "little" and gas is "very low" then the alarm is "potential danger".

Figure 1 is the simulation where the data from fire, smoke, and gas are manually input. The system will then decide whether the data input meets one of the 125 fuzzy rules. The range for the variable of fire is between $1 \mathrm{~nm}$ and $1100 \mathrm{~nm}$, the range for variable smoke is between 0 ppm and 10.000 while the range variable of gas is between 0 ppm and 2000 ppm. Figure 3 is an example where we input the value for fire, smoke and gas are $814 \mathrm{~nm}, 306 \mathrm{ppm}, 177 \mathrm{ppm}$ respectively. As the result, the alarm has yellow color or potential danger and, the water and exhaust fan sensors are active.

The next step is implementing it by using sensors. Arduino flame sensor and MQ2 smoke and gas sensor were used in order to obtain the dataset each of the input variables. To produce the buzzer alarm and the LED based on the result, the program must be connected with the Arduino IDE. The system is developed using PHP and the data received from the sensor will be used to determine whether the fire is not dangerous, potentially dangerous or dangerous.

Figure 2 shows the architecture of fire hazard system containing the three sensors (flame, smoke, and gas) that connected with Arduino. LED shows the light indicator which is giving a red if its dangerous condition, yellow if potential danger and green mean not dangerous.

Figure 3 shows the tools that detect the fire, smoke, and gas. After one of the fuzzy rules has been chosen according to the data received the output will be the sound of the buzzer and the color of LED. If the result is not dangerous then the LED will remain green and the sound of the buzzer is in a slower mode. If the result is potentially dangerous then the LED will turn yellow and the sound of the buzzer is in medium mode. Lastly, the color of the LED will turn red and the buzzer is in faster mode showing that the situation could be dangerous.

Table 2 shows the specification of flame sensor consisting with the symbol, value, and the unit in flame sensor detection. 


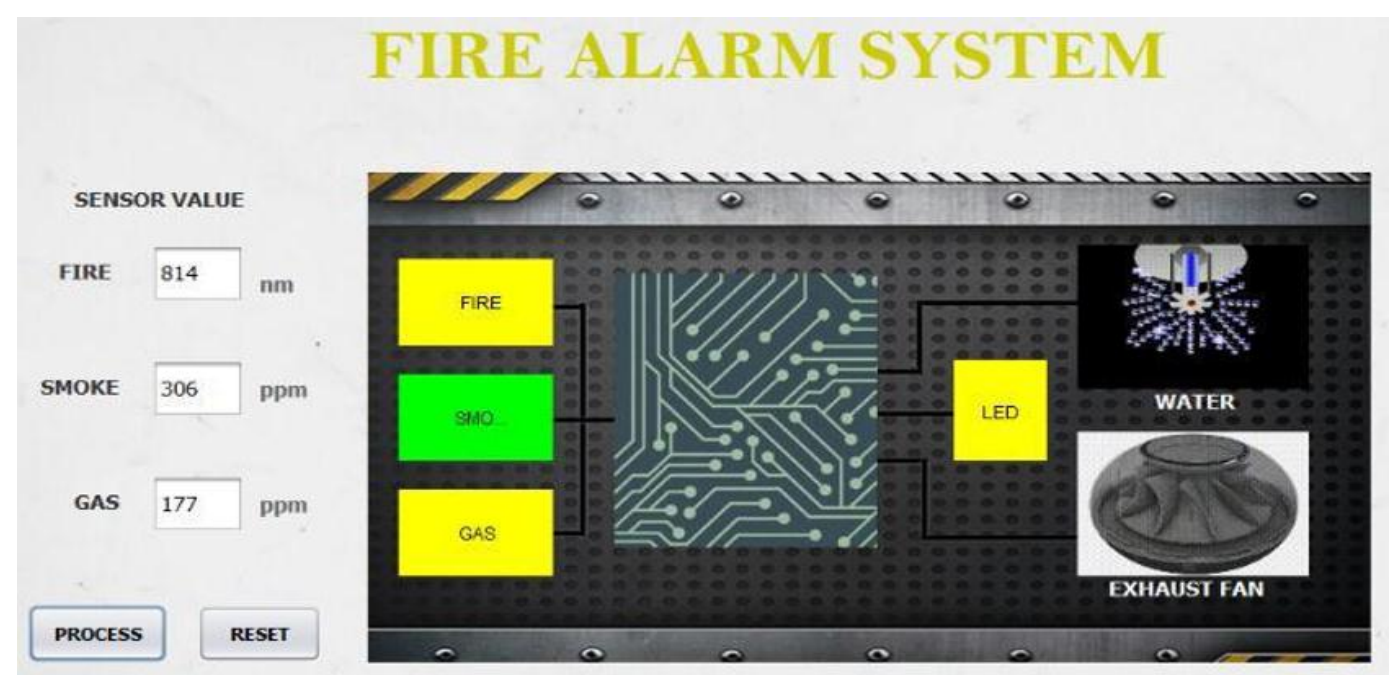

Figure 1 GUI of fire hazard detection

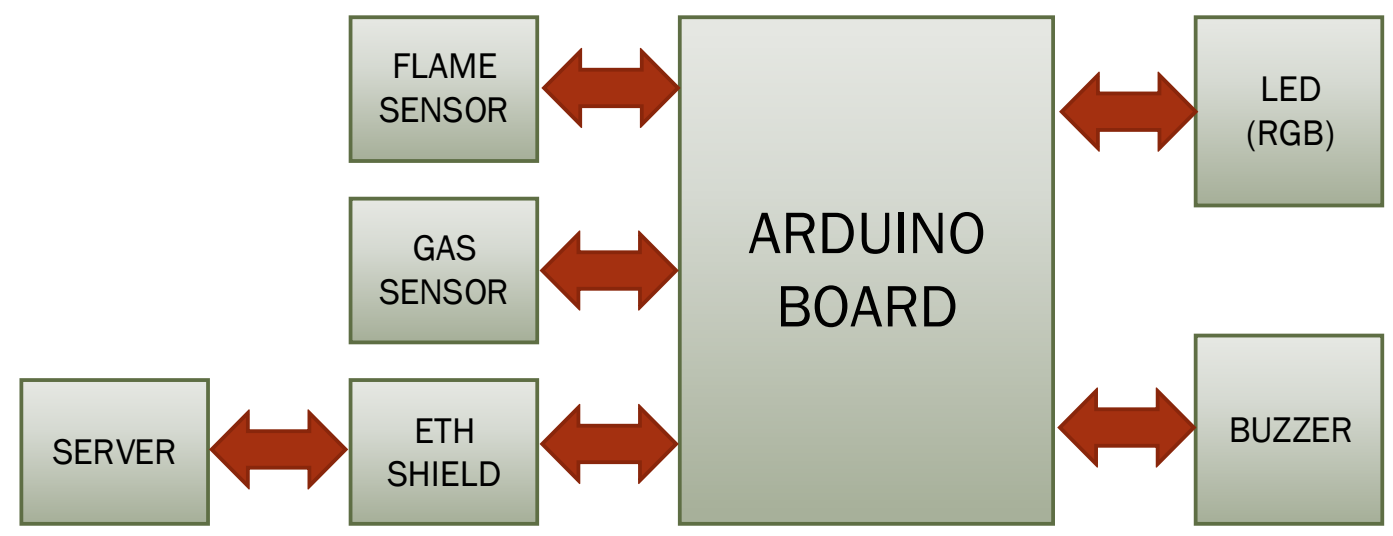

Figure 2 The architecture of fire hazard system

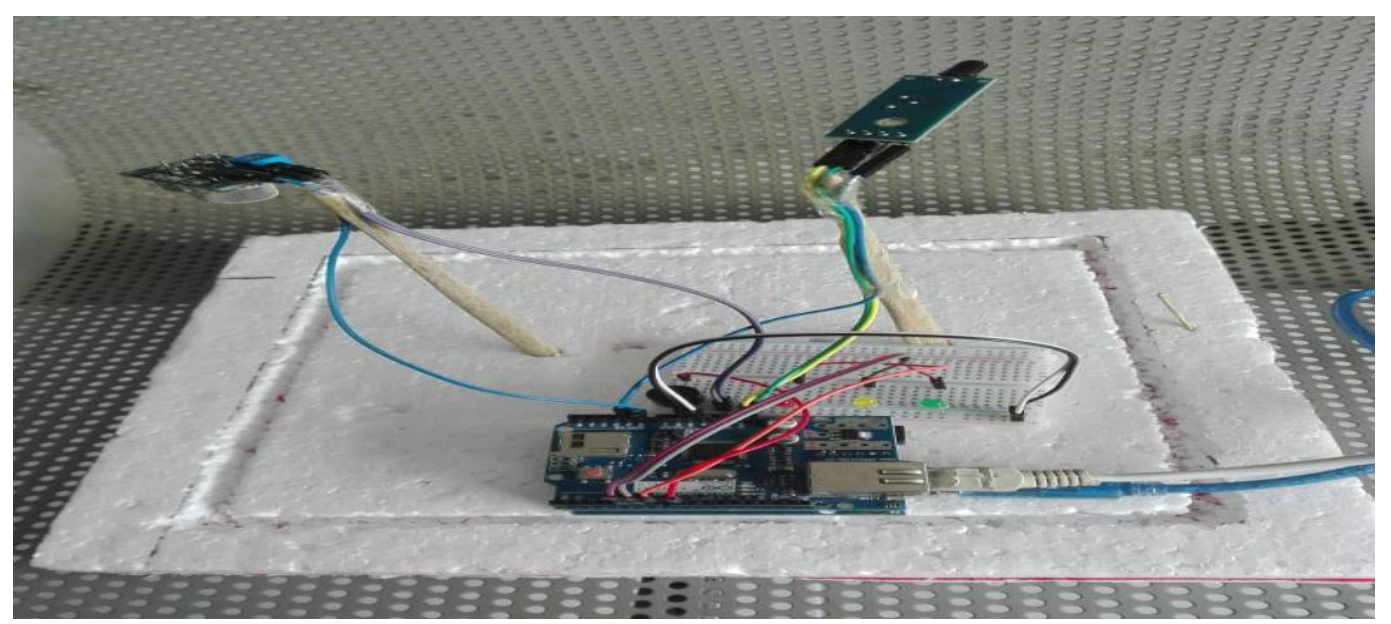

Figure 3 Prototype of fire hazard detection using Arduino 
Ause Labellapansa et al.

Table 2 Specification of the flame sensor

\begin{tabular}{llll}
\hline Item & Symbol & Value & Unit \\
\hline DC Forward Current & $\mathrm{I}_{\mathrm{F}}$ & 100 & $\mathrm{~mA}$ \\
Pulse Forward Current* & $\mathrm{IFP}$ & 1000 & $\mathrm{~mA}$ \\
Reverse Voltage & $\mathrm{V}_{\mathrm{R}}$ & 5 & $\mathrm{~V}$ \\
Power Dissipation & $\mathrm{P}_{\mathrm{D}}$ & 180 & $\mathrm{~mW}$ \\
Operating Temperature & Topr & $-30 \sim+85$ & ${ }^{\circ} \mathrm{C}$ \\
Storage Temperature & Tstg & $-40 \sim+100$ & ${ }^{\circ} \mathrm{C}$ \\
Lead Soldering Temperature & Tsol & $260^{\circ} \mathrm{C} / 5 \mathrm{sec}$ & - \\
\hline
\end{tabular}

\section{Results and discussions}

Table 3 shows the result of the fire alarm system after five trials were conducted. Each trial obtained 10 outputs for every input variable. There were 6 errors outputs in this fire alarm system which is calculated as below:

Fire alarm output $=\frac{6}{50} \times 100=12 \%$

Table 3 Result of fire alarm system

\begin{tabular}{|c|c|c|c|c|c|c|}
\hline \multicolumn{4}{|c|}{ Actual data } & \multicolumn{3}{|c|}{ Fire alarm system } \\
\hline Testing & Flame & Smoke & Gas & Alarm & Alarm & Result \\
\hline \multirow[t]{10}{*}{1} & 760 & 3098 & 1536 & Red & Yellow & Failed \\
\hline & 814 & 306 & 177 & Yellow & Yellow & Success \\
\hline & 886 & 844 & 433 & Yellow & Yellow & Success \\
\hline & 798 & 519 & 1374 & Red & Red & Success \\
\hline & 892 & 4745 & 38 & Yellow & Yellow & Success \\
\hline & 799 & 1211 & 71 & Red & Red & Success \\
\hline & 897 & 4625 & 571 & Red & Red & Success \\
\hline & 809 & 2643 & 1858 & Red & Red & Success \\
\hline & 765 & 9558 & 16 & Yellow & Yellow & Success \\
\hline & 897 & 6966 & 37 & Red & Red & Success \\
\hline \multirow[t]{10}{*}{2} & 793 & 7831 & 264 & Red & Red & Success \\
\hline & 811 & 9136 & 1127 & Red & Red & Success \\
\hline & 896 & 7593 & 1612 & Red & Red & Success \\
\hline & 944 & 329 & 50 & Yellow & Yellow & Success \\
\hline & 961 & 139 & 32 & Green & Yellow & Failed \\
\hline & 933 & 917 & 1644 & Red & Red & Success \\
\hline & 974 & 5220 & 367 & Red & Red & Success \\
\hline & 1021 & 387 & 213 & Yellow & Yellow & Success \\
\hline & 1026 & 315 & 29 & Green & Green & Success \\
\hline & 1028 & 8186 & 1606 & Yellow & Yellow & Success \\
\hline \multirow[t]{10}{*}{3} & 983 & 1762 & 197 & Yellow & Yellow & Success \\
\hline & 1033 & 6320 & 348 & Yellow & Yellow & Success \\
\hline & 1056 & 299 & 35 & Green & Yellow & Failed \\
\hline & 1074 & 483 & 1141 & Yellow & Yellow & Success \\
\hline & 1053 & 671 & 491 & Yellow & Yellow & Success \\
\hline & 707 & 225 & 30 & Green & Yellow & Failed \\
\hline & 1055 & 8698 & 27 & Yellow & Yellow & Success \\
\hline & 1054 & 2532 & 41 & Yellow & Yellow & Success \\
\hline & 1048 & 3870 & 171 & Yellow & Yellow & Success \\
\hline & 1063 & 9102 & 679 & Yellow & Yellow & Success \\
\hline
\end{tabular}


International Journal of Advanced Computer Research, Vol 9(44)

\begin{tabular}{lllllll}
\hline & Actual data & & & & Fire alarm system & \\
\hline Testing & Flame & Smoke & Gas & Alarm & Alarm & Result \\
\hline 4 & 59 & 4771 & 504 & Red & Yellow & Failed \\
& 420 & 4731 & 879 & Red & Yellow & Failed \\
& 1033 & 6320 & 348 & Yellow & Yellow & Success \\
& 931 & 7891 & 860 & Red & Red & Success \\
& 920 & 8797 & 888 & Red & Red & Success \\
& 1063 & 9102 & 679 & Yellow & Yellow & Success \\
& 1063 & 9102 & 679 & Yellow & Yellow & Success \\
& 1048 & 3870 & 171 & Yellow & Yellow & Success \\
& 920 & 8797 & 888 & Red & Red & Success \\
& 987 & 7891 & 887 & Red & Red & Success \\
\hline 5 & 1053 & 671 & 491 & Yellow & Yellow & Success \\
& 896 & 483 & 1141 & Yellow & Yellow & Success \\
& 944 & 671 & 491 & Yellow & Yellow & Success \\
& 961 & 329 & 35 & Yellow & Yellow & Success \\
& 933 & 139 & 1141 & Yellow & Yellow & Success \\
& 1053 & 671 & 491 & Yellow & Yellow & Success \\
& 1056 & 299 & 300 & Green & Green & Success \\
& 1074 & 483 & 270 & Yellow & Yellow & Success \\
& 1053 & 671 & 198 & Yellow & Yellow & Success \\
& 707 & 225 & 56 & Yellow & Yellow & Success \\
\hline
\end{tabular}

The results obtained from the fire alarm system using Arduino Uno indicates there were $12 \%$ errors and it is still considered as small. Therefore, it shows that this method is suitable to be used in determining the fire alarm system as the output shows $88 \%$ accuracy of the results.

\section{Conclusions and recommendations}

As the conclusion, the system is developed to give a warning and caution to save people from fire threats. This system has reached our main objective which is activating the sensor and alarm. A vigorous result had obtained with the help of a fuzzy logic approach. The sensors will inform the value of fire, smoke and gas reading to the server for the administrator to analyses. Finally, the alarm will activate to alert everyone so that they can take precautionary actions. Based on the current system, this system can be upgraded by adding an exhaust fan and water sensor to be monitored to run an alarm that has multipurpose and can take early precautions of hazards that occurred. These steps are necessary to make a systematic and functional system in the future.

\section{Acknowledgment}

This research is partially supported by Universitas Islam Riau, Indonesia and Universiti Teknologi MARA, Malaysia.

\section{Conflicts of interest}

The authors have no conflicts of interest to declare.

\section{References}

[1] Department of Fire and Safety. http://www.jakartafire.net/statistic. Accessed 8 January 2019.

[2] Aditya A. Cigarette smoke detectors in the room using MQ-2 sensor and arduino uno microcontroller. Syntax: Jurnal Informatika. 2017; 6(1):37-46.

[3] Ramya V, Palaniappan B. Embedded system for hazardous gas detection and alerting. International Journal of Distributed and Parallel Systems. 2012; 3(3):287-300.

[4] Reddy M, Rao K. Fire accident detection and prevention monitoring system using wireless sensor network enabled android application. Indian Journal of Science and Technology. 2016; 9(17):1-5.

[5] Ananda N. Designing cigarette smoke detection tool in cigarette-based smoking room based on microcontroller (Doctoral Dissertation, Padang State Polytechnic).

[6] Jannah M. Designed to build a fire smoke detection device using the MQ-2 sensor based on arduino uno. 2017.

[7] Utomo BT, Saputra DS. Simulation of room pollution detection system using smoke sensors with notification via SMS (Short Message Service) and arduino based alarms. Asian Information Technology Scientific Journal. 2016; 10 (1): 56-68.

[8] Manihar SR, Dewagan KP, Rajpurohit J. Multiple gas analyzer and indicator. International Journal of Modern Engineering Research. 2012; 2(4):2753-5. 
[9] Klęsk P, Korzeń M, Cariow A, Cariowa G. A mobile gas detector with an Arduino microcontroller. International Journal of Computer Applications in Technology. 2015; 6(4):636-41.

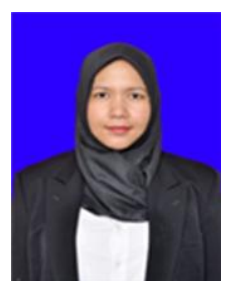

Ause Labellapansa earned her master degrees in Universitas Gadjah Mada (Artificial Intelligence) and Universitas Islam Indonesia (Medical informatics focused on Medical Imaging), Yogyakarta - Indonesia simultaneously from 2011 to early 2014. She is currently the head department of Informatics in Universitas Islam Riau (UIR) Indonesia. She has experienced several projects related to Artificial Intelligence especially on Rule-Based Reasoning \& CaseBased Reasoning. Her research interests are Expert System, Data Mining and Machine Learning.

Email: ause.labella@eng.uir.ac.id

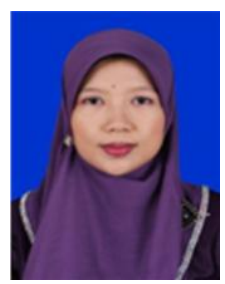

Nesi Syafitri received her Bachelor degree in Information System from Universitas Putra Indonesia "YPTK", Padang-Indonesia in 2003 and the master degree of Computer Science from Universitas Gadjah Mada in 2009. Her current position is Lecturer at the Faculty of Engineering, department of Informatics in Universitas Islam Riau (UIR), Indonesia. Her research interests are Fuzzy Logic, Data Mining and Machine Learning.

Email: nesisyafitri@eng.uir.ac.id

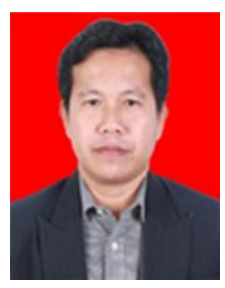

Evizal Abdul Kadir received his Master of Engineering and $\mathrm{PhD}$ in Wireless Communication at Faculty of Electrical Engineering, Universiti Teknologi Malaysia in 2008 and 2014 respectively. He is currently as Lecturer and Researcher in Islamic University of Riau (UIR) Indonesia as well as Director of research institute and community services and get promoted to Associate Professor. He has experience and worked in several companies that provide a system solution in Wireless Communication and Radio Frequency (RF) as well as Radio Frequency Identification (RFID), currently is continuing his research activities related to the Wireless Communication System, Antenna, Remote Sensing, RFID, Wireless Sensor Network (WSN), Wireless and Mobile Monitoring System and IoT.

Email: evizal@eng.uir.ac.id

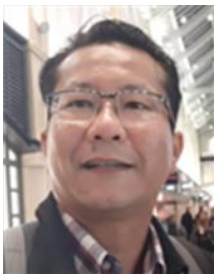

Rizauddin Saian received his degree of Bachelor of Science in Mathematics from Universiti Malaya, Malaysia in 1998 and the degree of Doctor of Philosophy from Universiti Utara Malaysia in 2013. His current position is Senior Lecturer at the Faculty of Computer and Mathematical Sciences, Universiti Teknologi MARA, Malaysia. His research interests are Data Mining and Machine Learning.

Email: rizauddin@uitm.edu.my

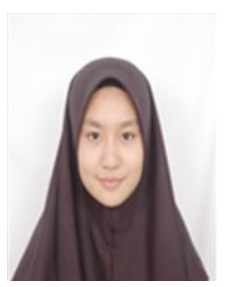

Aqilah Saibah Binti Abdul Rahman earned her Diploma in Computer Science from University Teknologi MARA, Arau Branch in 2019 and currently pursuing her bachelor's degree in computer science at University Teknologi MARA, Shah Alam. She has experienced several projects related to programming, especially on developing systems. Her research interests include Fuzzy Logic Approach using Arduino Uno to improvise the current fire alarm system which leads to the occurrence of false alarm because of inaccurate reading of fire, smoke and gas.

Email: aqilah.rahman01@gmail.com

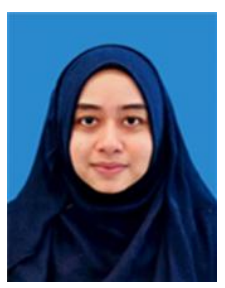

Marina Binti Ahmad received her Diploma in Computer Science from Universiti Teknologi MARA, Perlis Branch in 2019 andpursuingg her studies in Bachelor of Information System (HONS) Intelligent Systems Engineering at Universiti Teknologi MARA, Shah Alam. Her experienced more programming and security. Her interests more to programming on developing systems. Her research interests include Fuzzy Logic Approach using Arduino Uno to improvise the current fire alarm system which leads to the false alarm because of inaccurate reading of fire, smoke and gas.

Email: marinahmad98@gmail.com

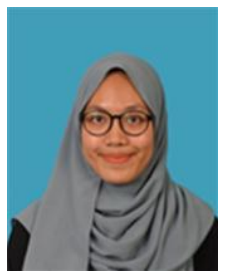

Siti Nurain Rusly received herDiploma inn Computer Science from Universiti Teknologi MAR, ,Perlis Branch in 2019. She is currently furthering her studies in Bachelor of Computer Science (Hons) at Universiti Teknologi MARA, Jasin Branch. Her research interests include the implementation of Fuzzy Logic Approach using Arduino Uno to improvise th ecurrent fire alarm system which leads to the occurrence off false alarm because an inaccurate reading of fire, smoke and gas.

Email: aqilah.rahman01@gmail.com 